\title{
Extended nonlinear waves in multidimensional dynamical lattices
}

\author{
Q.E. Hoq ${ }^{\text {a }}$, J. Gagnon ${ }^{\text {b }}$, P.G. Kevrekidis ${ }^{\text {b }}$, B.A. Malomed ${ }^{\text {c }}$, \\ D.J. Frantzeskakis ${ }^{\mathrm{d}}$, R. Carretero-González ${ }^{\mathrm{e}, *}$ \\ ${ }^{a}$ Department of Mathematics, Western New England College, Springfield, MA 01119, USA \\ ${ }^{\mathrm{b}}$ Department of Mathematics and Statistics, University of Massachusetts, Amherst, MA 01003-4515, USA \\ ${ }^{\mathrm{c}}$ Department of Physical Electronics, Faculty of Engineering, Tel Aviv University, Tel Aviv 69978, Israel \\ ${ }^{\mathrm{d}}$ Department of Physics, University of Athens, Panepistimiopolis, Zografos, Athens 15784, Greece \\ e Nonlinear Dynamical Systems Group, ${ }^{1}$ Computational Sciences Research Center, and Department of Mathematics and Statistics, \\ San Diego State University, San Diego, CA 92182-7720, USA
}

Available online 2 September 2009

\begin{abstract}
We explore spatially extended dynamical states in the discrete nonlinear Schrödinger lattice in two- and three-dimensions, starting from the anti-continuum limit. We first consider the "core" of the relevant states (either a two-dimensional "tile" or a three-dimensional "stone"), and examine its stability analytically. The predictions are corroborated by numerical results. When the core is stable, we propose a method allowing the extension of the structure to as many sites as may be desired. In this way, various patterns of excited sites can be formed. The stability of the full extended nonlinear structures is studied numerically, which yields instability thresholds for such structures, which are attained with the increase of the lattice coupling constant. Finally, in cases of instability, direct numerical simulations are used to elucidate the evolution of the pattern; it is found that, typically, the unstable extended nonlinear pattern breaks up in an oscillatory way, leading to "lattice turbulence".
\end{abstract}

(C) 2009 IMACS. Published by Elsevier B.V. All rights reserved.

PACS: $05.45 . \mathrm{Yv} ;$ 42.65.Tg; 03.75.Lm; 04.60.Nc

Keywords: Nonlinear lattices; Discrete solitons; Nonlinear Schrödinger equation

\section{Introduction}

In the past two decades, a large number of studies have been devoted to discrete nonlinear dynamical models. This work has been motivated by diverse physical settings, including nonlinear optics of waveguide arrays [6] and photonic crystal lattices [8], Bose-Einstein condensates (BECs) trapped in deep optical lattices [2], models of the DNA double strand [19], and others. Many theoretical developments along these experimentally relevant directions have been spurred by an increasing level of understanding of Hamiltonian models of dynamical lattices, starting from a prototypical lattice that arises in diverse ways in the above-mentioned settings, namely, the discrete nonlinear Schrödinger (DNLS) equation [12].

Another direction that has been increasingly popular in the last several years focuses on the studies of the so-called $X$-waves, i.e., quasi-linear extended excitations in higher dimensions [15,5] (the name is due to the fact that, in the

\footnotetext{
* Corresponding author.

E-mail address: carreter@ sciences.sdsu.edu (R. Carretero-González).

1 URL: http://nlds.sdsu.edu/.
} 
two-dimensional (2D) space, the shape of such a partly delocalized wave is in the form of letter X). Very recently, an experimental evidence of spontaneous formation of $X$-waves in waveguide arrays was reported [13].

It is at this juncture between extended X-wave-shaped excitations and DNLS equations that the present paper aims at contributing. In particular, elaborating on the earlier work of Ref. [11], we report results concerning the existence, stability and dynamics of extended nonlinear wave configurations in the multidimensional DNLS equation. As will be discussed in detail below, the DNLS equation admits an analysis of the so-called anti-continuum (AC) limit (the one in which lattice sites are decoupled), where purely nonlinear analogs of the $\mathrm{X}$-waves can be constructed. In that limit, the X-shape is not enforced by the elementary symmetry of the linear operator (d'Alembertian), as is the case in regular X-waves; rather, one can construct arbitrary configurations, but simple rules make it possible to predict the existence and stability of the patterns that are going to appear from the arbitrary ones existing at small nonzero values of the lattice coupling constant. In this way, relevant patterns can be selected from the variety of arbitrary ones. It is the structures of this sort that we aim to explore in the present paper.

Our starting point is the standard DNLS equation on the 2D or 3D lattice:

$$
i \dot{u}_{\mathbf{n}}=-\epsilon\left(\Delta_{2} u\right)_{\mathbf{n}}-\left|u_{\mathbf{n}}\right|^{2} u_{\mathbf{n}},
$$

where $u_{\mathbf{n}}$ is the complex lattice field, $\mathbf{n}$ is the vectorial lattice index, $\Delta_{2}$ is the standard discrete Laplacian, and $\epsilon$ is the intersite coupling. We use the overdot to represent the derivative with respect to the evolution variable, which could be the longitudinal coordinate $z$ in the case of optical waveguide arrays, or the time $t$ in BEC models.

Our aim is, in particular, to examine multi-legged extended nonlinear structures (ENSs) in 2D and 3D discrete media via the identification of appropriate solutions to Eq. (1). The stability of the basic building blocks of the solutions ("tiles" and "stones", in the 2D and 3D cases, respectively), will be explored too in an analytical form, and will be compared to results of the numerical linear-stability analysis. For the structures whose "tiles" and "stones" will be found to be stable, we will examine how to prolong them to an arbitrary length, with the objective to construct ENSs, by appending effectively one-dimensional legs with alternating phases between adjacent blocks (which turns out to be necessary for the stability of the eventual ENS). Several varieties of the extended solutions will be thus constructed, including $\mathrm{X}$-waves, Y-waves, Z-waves, among others, in 2D; they are categorized by the number of legs they feature. Arguably, the simplest example will be a single-leg structure (referred to, hereafter, as configuration 1; see Fig. 1), whose tile consists of two sites with the phase shift of $\pi$ between them (the "twisted mode"). Similarly, we will consider 3D structures whose cores (stones) are structurally stable.

The ENS patterns that we hereby construct are based on different combinations of lines consisting of contiguous active sites that have alternating signs.

In the absence of any periodicity in the supporting medium (cf. absence of a periodic external potential) such ENS patterns clearly do no exist in the 2D and 3D continuum limit (which corresponds to $\epsilon \rightarrow \infty$ ) since not even a single twisted localized mode (a dipole with contiguous sites of opposite signs) survives in the continuum limit; see Ref. [1] for a detailed account of the termination scenaria, typically through saddle-node or pitchfork bifurcations, for the various
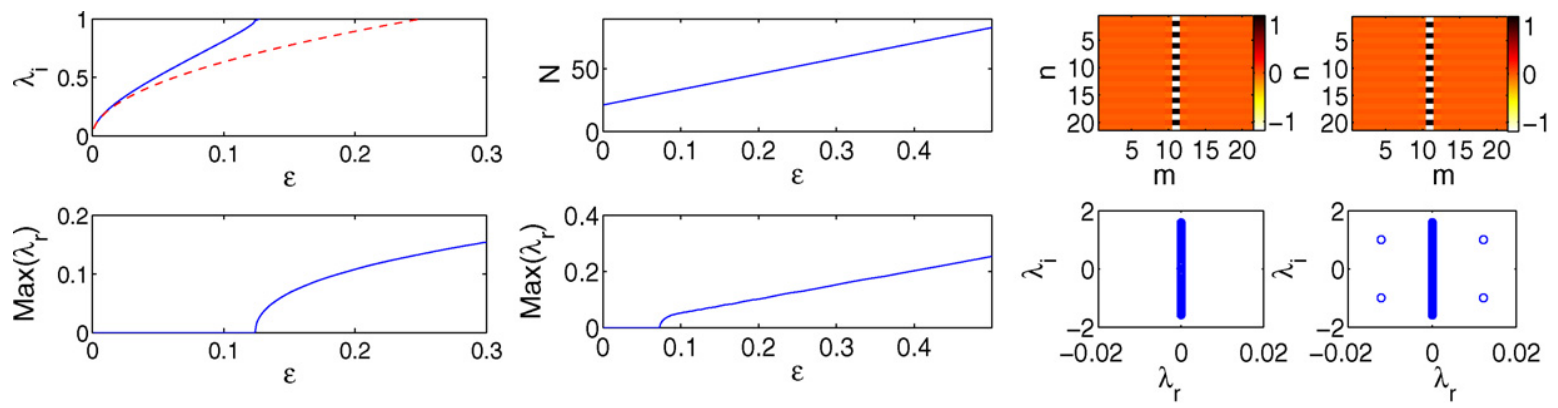

Fig. 1. The left panels relate to the tile from which the single-leg structure is built. The top one shows the relevant linear stability eigenvalue for the tile and compares the numerical results (solid lines) with the analytical prediction (dashed line). The bottom left panel shows the largest instability growth rate versus $\epsilon$ for the twisted tile. Note that, in this panel, Max $\left(\lambda_{r}\right)$ leaves the horizontal axis when the stability eigenvalue (in the top left panel) attains the value 1 (which happens between $\epsilon=0.124$ and 0.125 ). The second column panels show the norm $N$ and largest instability growth rate versus $\epsilon$ for the single-leg ENS pattern. Finally, the group of the four right panels display the single-leg configuration crossing the threshold from being stable $(\epsilon=0.073)$, on the left, to unstable $(\epsilon=0.074)$, on the right. 
branches of basic discrete solutions as the coupling parameter is increased. For example, it is worthwhile to note that in the 1D DNLS equation, only the single-site or two-site in-phase initial excitation will survive in the continuation for all values of $\epsilon$ up to the continuum limit of $\epsilon \rightarrow \infty$, where they will both degenerate into the continuum bright soliton solution of the integrable 1D NLS model. On the other hand, if a continuous medium is equipped with a periodicity, provided, for example, by an external periodic potential, it is enabled to support certain structures that do not exist or that are unstable in the homogenous case; these structures can often be predicted by a corresponding discrete model, whereby each cell of the continuum periodic model is represented by a single site. For instance, quasi-discrete vortex solitons [16,7], quasi-discrete dipoles (twisted localized modes) and quadrupoles [20], and even trains of bright [4] or gap solitons [14] can be created in such optically induced photonic lattices.

By continuation from the AC limit it is possible to construct ENS patterns for finite values of the coupling constant $\epsilon$. Typically, as the coupling is increased from the AC limit, these ENS lose their stability well before their respective branch terminates as one approaches the continuum limit as described above.

We will therefore identify the stability limits, in terms of $\epsilon$, for each of the proposed structures (as concerns both their tiles/stones and the entire ENS configurations). In the case when ENSs are unstable, we will also carry out direct simulations of the instability development. Typically, oscillatory growth of the instability will be demonstrated, associated with a quartet of eigenvalues that account for the instability in terms of the linear spectrum of small perturbations around the unstable ENS. Eventually, this evolution leads to quasi-turbulent dynamics of the lattice, without an apparent robust asymptotic state.

The paper is organized as follows: first, in Section 2, we present analytical results and proceed by obtaining numerical existence and (linear-)stability conditions for various configurations. In Section 3, we present the bifurcation analysis of the relevant structures, while in Section 4, we report results of direct simulations pertaining to unstable patterns. Finally, Section 5 concludes the paper.

\section{Theory}

We are interested in stationary solutions to Eq. (1) in the form of $u_{\mathbf{n}}=\exp (i \Lambda t) \phi_{\mathbf{n}}$, where $\Lambda$ is the propagation constant in optics or (minus) the chemical potential in BECs. The spatial profile $\phi_{\mathbf{n}}$ is governed by the following steady-state equation:

$$
G\left(v_{\mathbf{n}}, \epsilon\right) \equiv \Lambda \phi_{\mathbf{n}}-\epsilon \Delta_{2} \phi_{\mathbf{n}}-\left|\phi_{\mathbf{n}}\right|^{2} \phi_{\mathbf{n}}=0
$$

In the AC (anti-continuum) limit, $\epsilon=0$, solutions to Eq. (2) are $\phi_{\mathbf{n}}=0$ or $\phi_{\mathbf{n}}=\sqrt{\Lambda} e^{i \theta_{\mathbf{n}}}$, where $\theta_{\mathbf{n}}$ is an arbitrary phase. Using the scaling invariance of Eq. (2), we set $\Lambda=1$ in what follows.

From the Lyapunov Reduction Theorem $[9,17,18]$ or from a direct calculation, ${ }^{2}$ we can obtain the following "solvability" condition:

$$
2 i g_{\mathbf{n}}(\theta, \epsilon) \equiv \epsilon e^{-i \theta_{\mathbf{n}}} \Delta_{2} \phi_{\mathbf{n}}-\epsilon e^{i \theta_{\mathbf{n}}}\left(\Delta_{2} \phi_{\mathbf{n}}^{*}\right)=0
$$

for the persistence of the solution, at each excited site, for infinitely small $\epsilon$ different from zero (in other words, for the continuability of the solution beyond the AC limit). This condition can then be readily implemented, once the profile of a particular configuration is known in the AC limit. Typically (although not exclusively) in what follows, we will deal with structures having $\theta_{\mathbf{n}}=0, \pi$.

Once the continuation of the structure to $\epsilon>0$ is confirmed, it is necessary to examine the linear stability of the corresponding stationary solution. To that effect, we use the perturbation ansatz:

$$
u_{\mathbf{n}}=e^{i \Lambda t}\left(\phi_{\mathbf{n}}+\delta a_{\mathbf{n}} e^{i \omega t}+\delta b_{\mathbf{n}} e^{-i \omega^{*} t}\right)
$$

\footnotetext{
2 The direct calculation consist of multiplying Eq. (2) by $\phi_{n}^{*}$ (which in the AC limit is $e^{-i \theta_{n}}$ ) and the conjugate of Eq. (2) by $\phi_{n}$ and subtracting the two.
} 
where $\delta$ is a formal small parameter. Substitution of Eq. (4) into Eq. (1) yields the following system of the linearized equations:

$$
\begin{aligned}
& -\omega a_{\mathbf{n}}=-\epsilon \Delta_{2} a_{\mathbf{n}}+\Lambda a_{\mathbf{n}}-2\left|\phi_{\mathbf{n}}\right|^{2} a_{\mathbf{n}}-\phi_{\mathbf{n}}^{2} b_{\mathbf{n}}^{*}, \\
& \omega^{*} b_{\mathbf{n}}=-\epsilon \Delta_{2} b_{\mathbf{n}}+\Lambda b_{\mathbf{n}}-2\left|\phi_{\mathbf{n}}\right|^{2} b_{\mathbf{n}}-\phi_{\mathbf{n}}^{2} a_{\mathbf{n}}^{*} .
\end{aligned}
$$

This system can be rewritten as

$$
\sigma \mathcal{H} \psi=i \lambda \psi
$$

where $\sigma$ is the matrix consisting of $2 \times 2$ blocks:

$$
\sigma_{n, m}=\left(\begin{array}{cc}
1 & 0 \\
0 & -1
\end{array}\right) \delta_{n, m}
$$

$\lambda=i \omega$, and $\psi=\left(a_{\mathbf{n}}, b_{\mathbf{n}}^{*}\right)^{T}$ (with superscript $T$ standing for transpose). The linearization operator corresponding to the perturbation of (1) around solutions of (2) is given by

$$
\mathcal{H}_{n}=\left(\begin{array}{cc}
\Lambda-2\left|\phi_{\mathbf{n}}\right|^{2} & -\phi_{\mathbf{n}}^{2} \\
-\left(\phi_{\mathbf{n}}^{*}\right)^{2} & \Lambda-2\left|\phi_{\mathbf{n}}\right|^{2}
\end{array}\right)-\epsilon \Delta_{2}\left(\begin{array}{ll}
1 & 0 \\
0 & 1
\end{array}\right) .
$$

In what follows, we will fix $\Lambda=1$ without loss of generality.

A remarkable feature, shown in Ref. [17], is that eigenvalues $\gamma$ of the Jacobian:

$$
\mathcal{M}_{i, j}=\frac{\partial g_{i}}{\partial \theta_{j}},
$$

are related to the underlying stability eigenvalues $\lambda$ by a simple expression, i.e., $\lambda=\sqrt{2 \epsilon \gamma}+O(\epsilon)$.

\section{Bifurcation analysis}

In this section we report numerical results for the existence and stability of the ENS-type solutions. We obtain the relevant configurations numerically, starting from the AC limit, and using a fixed-point iteration for $\epsilon \neq 0$, to continue the solution to finite values of $\epsilon$. Upon obtaining the solution to a desired numerical precision (typically $10^{-8}$ ), we examine its linear stability by numerically solving the eigenvalue problem based on Eq. (5). In fact, the main objective of the latter stage of the analysis will be to look for bifurcations of the eigenvalues that account for the destabilization of the stationary solutions at finite $\epsilon$. Since our presentation of these results will be uniform for all the solutions, it is necessary to explain the relevant format. Fig. 1 shows panels related to the stability of the twisted mode (the corresponding tile), as well as the ENS of the configuration of type 1, as defined above. The two left panels will always be used to show the imaginary part $\left(\lambda_{i}\right)$ and largest real part $\left(\operatorname{Max}\left(\lambda_{r}\right)\right)$ of the eigenvalues, as functions of $\epsilon$, for the tile. Notice that the largest real part among the eigenvalues is tantamount to the leading growth rate of the instability of the respective configuration. The two panels in the second column will always show the norm $\left(N=\sum_{n} \phi_{n}^{2}\right)$ and the maximum instability growth rate for the full configuration (i.e., the ENS per se). Finally, the group of the four right panels demonstrate contour plots of the spatial profile $\phi_{\mathbf{n}}$ of the given configuration, and the corresponding spectral planes of the linearization eigenvalues, $\left(\lambda_{r}, \lambda_{i}\right)$, for values of the coupling strength just before (left) and immediately after (right) the instability threshold. In the case of configuration 1, these plots are displayed for $\epsilon=0.073$ and 0.074 , on the left and right, respectively. The change in the spectral plane shows that the stability threshold is located between these values, due to the appearance of a complex eigenvalue quartet (indicating an oscillatory instability). Similar figures follow for other species as well.

For the twisted mode, the Jacobian (6), with $g_{j}=\sin \left(\theta_{j}-\theta_{j+1}\right)+\sin \left(\theta_{j}-\theta_{j-1}\right)$, yields the stability eigenvalues $\lambda= \pm \sqrt{2 \gamma}= \pm 2 \sqrt{\epsilon} i$. Fig. 1 shows results related to the stability of the twisted mode, as well as the respective ENS.

The two-legged ENSs that we consider here maybe classified as $X$-waves (configuration 2a), $Y$-waves (configuration $2 \mathrm{~b}$ ), and skewed $X$-waves (configuration 2c). Stable $\mathrm{X}$-shaped configurations can be built out of tiles which in the AC 
limit look like:

$$
\left(\begin{array}{ccc}
1 & 0 & -1 \\
0 & 0 & 0 \\
-1 & 0 & 1
\end{array}\right)
$$

The corresponding Jacobian $\mathcal{M}_{i, j}$ is given by Eq. (6), where $j=1,2,3,4$. This tile gives rise to the pair of eigenvalues $\lambda= \pm 2 \sqrt{2} \epsilon i$, and the double one, $\lambda= \pm 2 \epsilon i$.

Note that an X-configuration can also be built from the tile:

$$
\left(\begin{array}{ccc}
1 & 0 & 1 \\
0 & -1 & 0 \\
1 & 0 & 1
\end{array}\right)
$$

However, this one gives rise to an unstable eigenvalue pair, $\lambda= \pm 2 \epsilon$.

In addition, Y-shaped and skewed $\mathrm{X}$-shaped configurations are built, respectively, of tiles:

$$
\left(\begin{array}{ccc}
1 & 0 & -1 \\
0 & 0 & 0 \\
0 & 0 & 1
\end{array}\right), \quad\left(\begin{array}{ccc}
0 & -1 & 1 \\
0 & 1 & 0 \\
1 & -1 & 0
\end{array}\right) .
$$

The Y-tile has the same form of the Jacobian as configuration $2 \mathrm{a}$ with $j=1,2,3$. The eigenvalues for this case are $\lambda= \pm \sqrt{2} \epsilon i$ and $\lambda= \pm \sqrt{6} \epsilon i$. The Jacobian of the skewed X-tile is given by Eq. (6) with

$$
\begin{aligned}
& g_{j}=\sin \left(\theta_{j}-\theta_{j+1}\right)+\sin \left(\theta_{j}-\theta_{j-1}\right), \quad j=1,2,3, \\
& g_{0}=\sin \left(\theta_{0}-\theta_{1}\right), g_{4}=\sin \left(\theta_{4}-\theta_{3}\right),
\end{aligned}
$$

and yields eigenvalues $\lambda= \pm 0.874 \sqrt{\epsilon} i, \lambda= \pm 1.662 \sqrt{\epsilon} i, \lambda= \pm 2.288 \sqrt{\epsilon} i, \lambda= \pm 2.690 \sqrt{\epsilon} i$. Figs. 2-4 illustrate properties of the $\mathrm{X}, \mathrm{Y}$ and skewed $\mathrm{X}$-waves in a format similar to the case of the configuration 1.

Next, we consider two stable three-legged ENS's, named configurations $3 \mathrm{a}$ and $3 \mathrm{~b}$, based on tiles:

$$
\left(\begin{array}{ccc}
1 & -1 & 1 \\
-1 & 1 & -1 \\
1 & -1 & 1
\end{array}\right) \text { and }\left(\begin{array}{ccc}
1 & -1 & 1 \\
0 & 1 & 0 \\
-1 & 0 & 0
\end{array}\right) \text {, }
$$

respectively. The Jacobian for the former one, 3a, is built of elements:

$$
\begin{aligned}
& g_{j}=\sin \left(\theta_{j}-\theta_{j+1}\right)+\sin \left(\theta_{j}-\theta_{j-1}\right), \quad j=1,3,5,7, \\
& g_{j}=\sin \left(\theta_{j}-\theta_{j+1}\right)+\sin \left(\theta_{j}-\theta_{j-1}\right)+\sin \left(\theta_{j}-\theta_{0}\right), \quad j=2,4,6,8, \\
& g_{0}=\sin \left(\theta_{0}-\theta_{2}\right)+\sin \left(\theta_{0}-\theta_{4}\right)+\sin \left(\theta_{0}-\theta_{6}\right)+\sin \left(\theta_{0}-\theta_{8}\right) .
\end{aligned}
$$
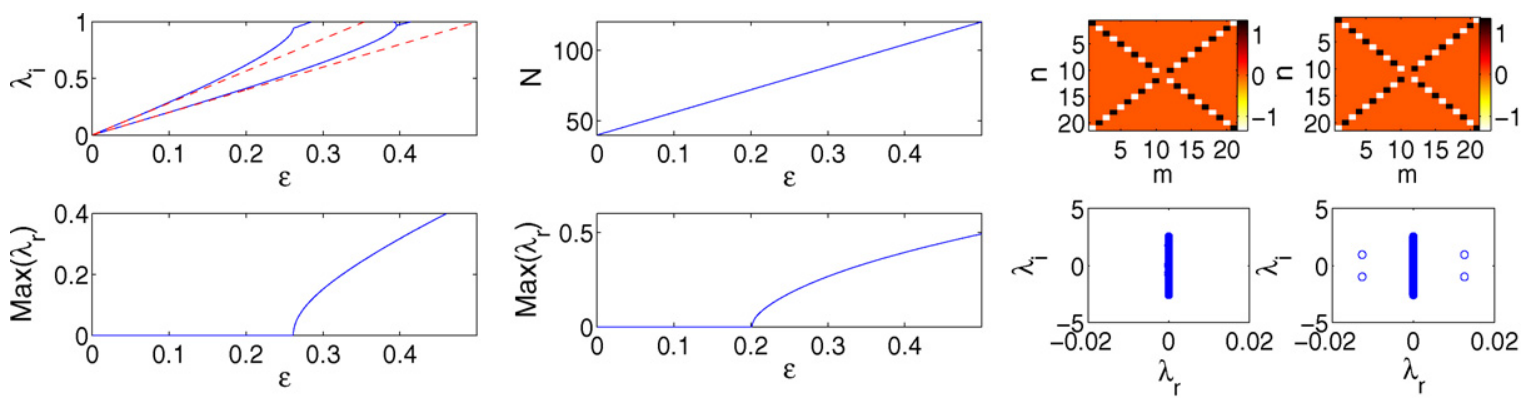

Fig. 2. This series of panels shows the same features for the $\mathrm{X}$-wave, named configuration 2a, as for configuration 1 in Fig. 1. The layout here is the same as in Fig. 1. The left panels relate to the tile from which the X-pattern is built, while the middle column panels show the norm and largest instability growth versus $\epsilon$ for the X-wave ENS pattern. The four right-column panels display the X-wave crossing the threshold from being stable (at $\epsilon=0.201$ ), on the left, to unstable (at $\epsilon=0.202$ ), on the right. 

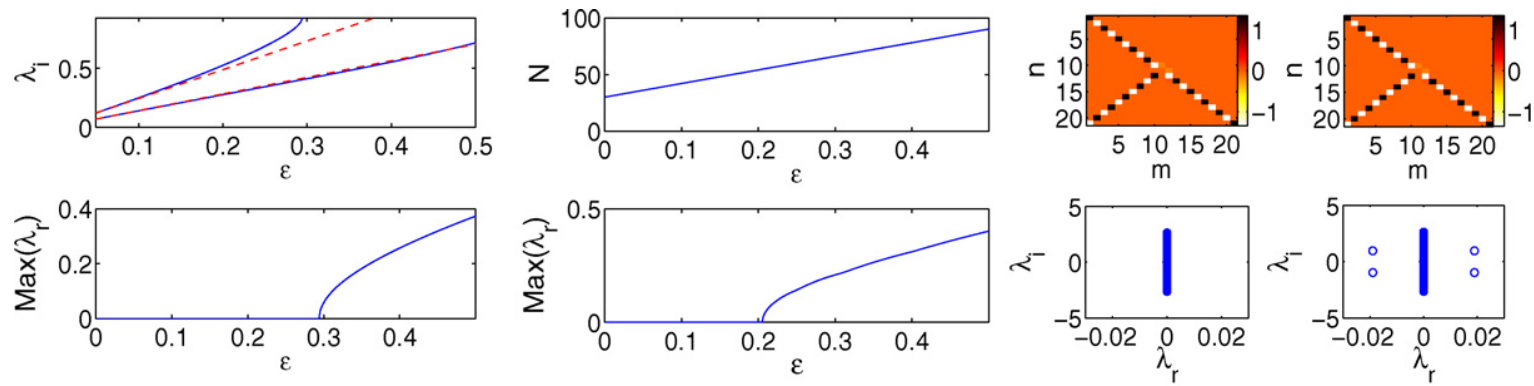

Fig. 3. This series of panels for the Y-wave, configuration $2 b$. The layout is the same as in Fig. 1. The four right panels display the Y-wave crossing the threshold from being stable (at $\epsilon=0.205$ ), on the left, to unstable (at $\epsilon=0.206$ ), on the right.
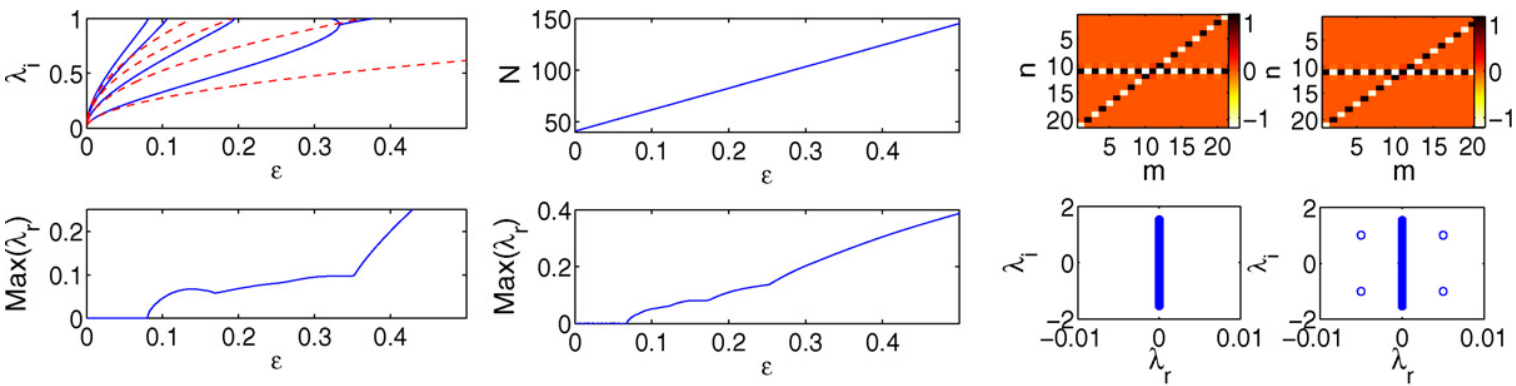

Fig. 4. Panels for the skewed X-wave, configuration 2c. This wave becomes unstable between points $\epsilon=0.067$ and 0.068 .

It generates three pairs of double eigenvalues, $\lambda= \pm \sqrt{2 \epsilon} i, \lambda= \pm \sqrt{6 \epsilon} i, \lambda= \pm \sqrt{8 \epsilon} i$, and two pairs of single eigenvalues, $\lambda= \pm 2 \sqrt{\epsilon} i, \lambda= \pm \sqrt{12 \epsilon} i$. The Jacobian for the latter configuration, $3 b$, is constructed via Eq. (6) from the following elements:

$$
\begin{aligned}
& g_{j}=\sin \left(\theta_{j}-\theta_{2}\right), \quad j=1,3,4, \\
& g_{j}=\sin \left(\theta_{2}-\theta_{1}\right)+\sin \left(\theta_{2}-\theta_{3}\right)+\sin \left(\theta_{2}-\theta_{4}\right), \quad j=2,
\end{aligned}
$$

and generates the following eigenvalues: a double one, $\lambda= \pm \sqrt{2 \epsilon} i$, and the singlet, $\lambda= \pm \sqrt{8 \epsilon} i$. Due to its shape, the ENS associated with configuration $3 \mathrm{~b}$ is also referred to as a Z-wave. Configurations $3 \mathrm{a}$ and $3 \mathrm{~b}$ are illustrated, respectively, in Figs. 5 and 6.

Let us now examine two cases of three-dimensional ENSs, to be called configurations $4 \mathrm{a}$ and $4 \mathrm{~b}$. The first of these has its core constructed from the same tile as $3 \mathrm{a}$, by attaching two -1 values to the sites on either side of the middle 1 along the third direction. This stone has the following single eigenvalues: $\lambda= \pm 1.248 \sqrt{\epsilon} i, \lambda= \pm 2 \sqrt{\epsilon} i, \lambda= \pm \sqrt{6 \epsilon} i, \lambda=$ $\pm 2.763 \sqrt{\epsilon} i, \lambda= \pm 3.848 \sqrt{\epsilon} i$, and double and triple eigenvalues $\lambda= \pm \sqrt{8 \epsilon} i$ and $\lambda= \pm \sqrt{2 \epsilon} i$, respectively.

Configuration $4 \mathrm{~b}$ is based on a stone with a diamond-like shape [3] with six sites, i.e., an out-of-phase quadrupole with alternating phases $(0, \pi, 0, \pi)$ in the plane and two sites with phases $\pi / 2$ and $3 \pi / 2$ outside the plane. This structure
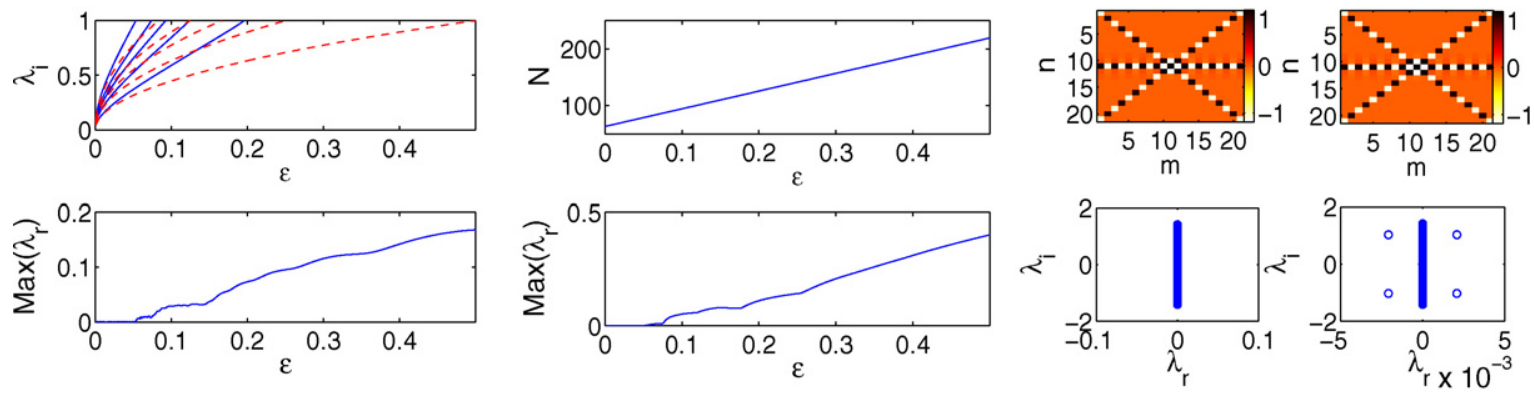

Fig. 5. Configuration 3a becomes unstable between points $\epsilon=0.052$ and 0.053 . 

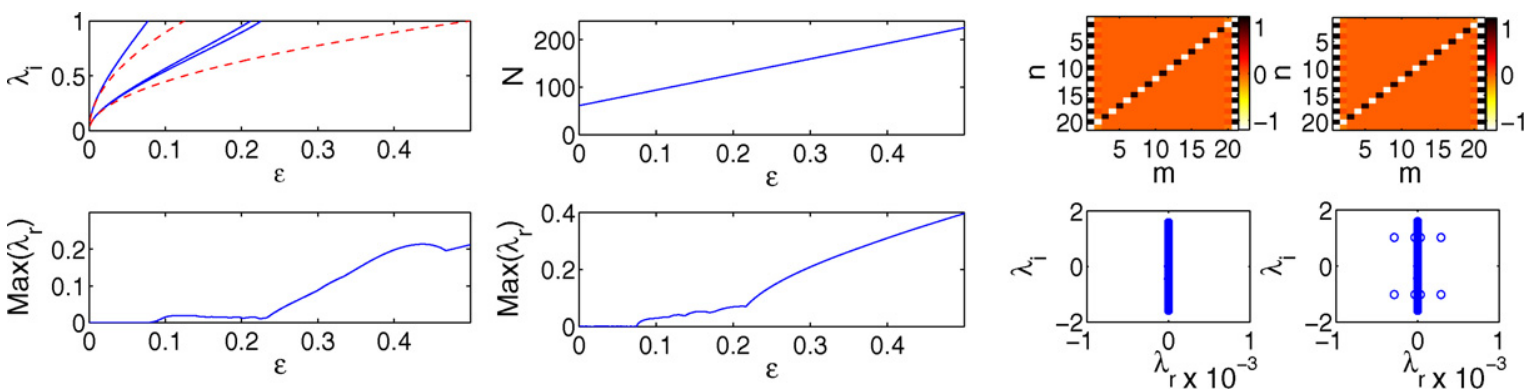

Fig. 6. Configuration 3b (Z-waves) becomes unstable between points $\epsilon=0.074$ and 0.075 .

has triple and single eigenvalues, $\lambda= \pm 2 \epsilon i$ and $\lambda= \pm 4 \epsilon i$, respectively, and a higher-order one, $\mathcal{O}\left(\epsilon^{2}\right)$. The respective 3D ENS are shown in Figs. 7 and 8.

Finally, since we are dealing with extended structures, the boundary effects due to lattice size must be monitored. In Figs. 9 and 10 we show the effects of the lattice size on the most unstable eigenvalue. The size of the lattice is taken as $N \times N$ for the 2D cases and $N \times N \times N$ for the 3D cases. As it can be observed from the figures, the behavior seems to converge for lattice sizes with $N \geq 11$. It is interesting to note that smaller lattice sizes increase the stability regions for the depicted configurations. This is natural since we are using zero boundary conditions that help suppress instabilities. Another interesting effect is that for some configurations, and small lattice sizes, extra stability regions are created by means of small stability windows [cf. Z-wave 2D pattern (right panel in Fig. 9) and, more notably, the 3D configuration 4a (left panel of Fig. 10)]. This is an effect similar to what is reported in Ref. [10] and is caused by the
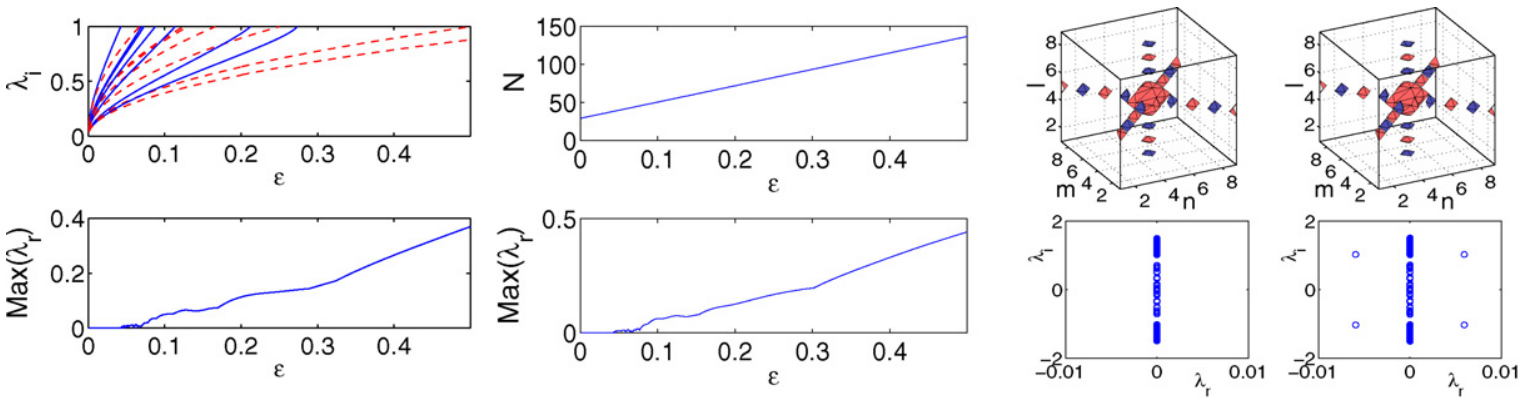

Fig. 7. These panels correspond to the three-dimensional configuration $4 \mathrm{a}$, which becomes unstable between points $\epsilon=0.043$ and 0.044 . These 3D, real, configurations are depicted using iso-level contours with level contours corresponding to $\operatorname{Re}\left(u_{m, n, l}\right)= \pm 0.5 \mathrm{Max}\left(\left|u_{m, n, l}\right|\right)$, which are shown in blue and red (dark gray and gray, in the black-and-white version), respectively. (For interpretation of the references to color in this figure legend, the reader is referred to the web version of the article.)
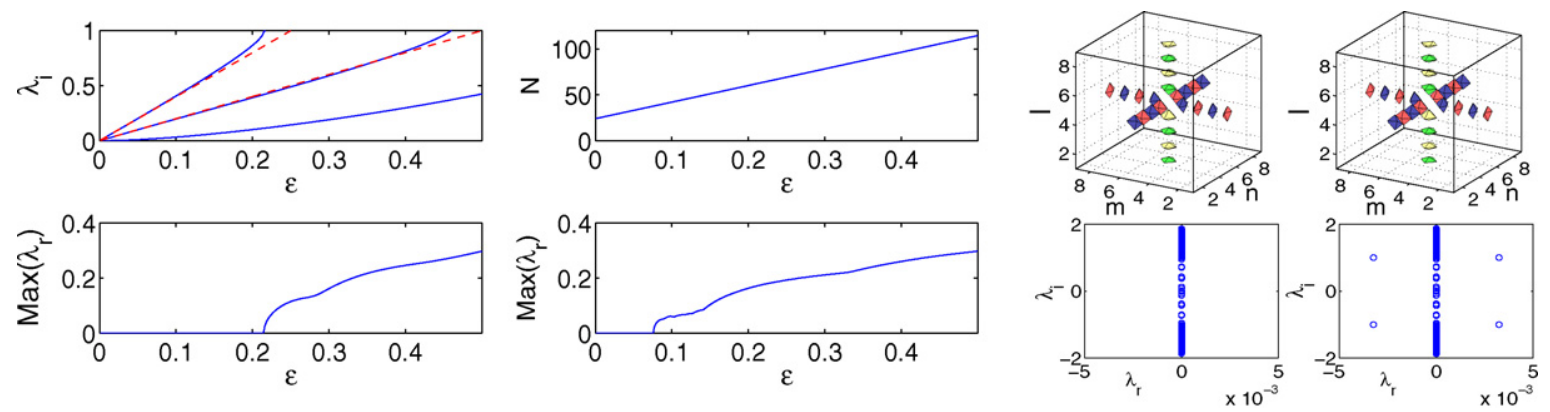

Fig. 8. This series of panels correspond to the three-dimensional configuration $4 \mathrm{~b}$. This state becomes unstable between points $\epsilon=0.075$ and 0.076. The 3D configurations are depicted using iso-level contours as explained in Fig. 7 for the real part and by imaginary contours, $\operatorname{Im}\left(u_{m, n, l}\right)=$ $\pm 0.5 \mathrm{Max}\left(\left|u_{m, n, l}\right|\right)$, shown in green and yellow (light and very light gray, in the black-and-white version) hues, respectively. (For interpretation of the references to color in this figure legend, the reader is referred to the web version of the article.) 

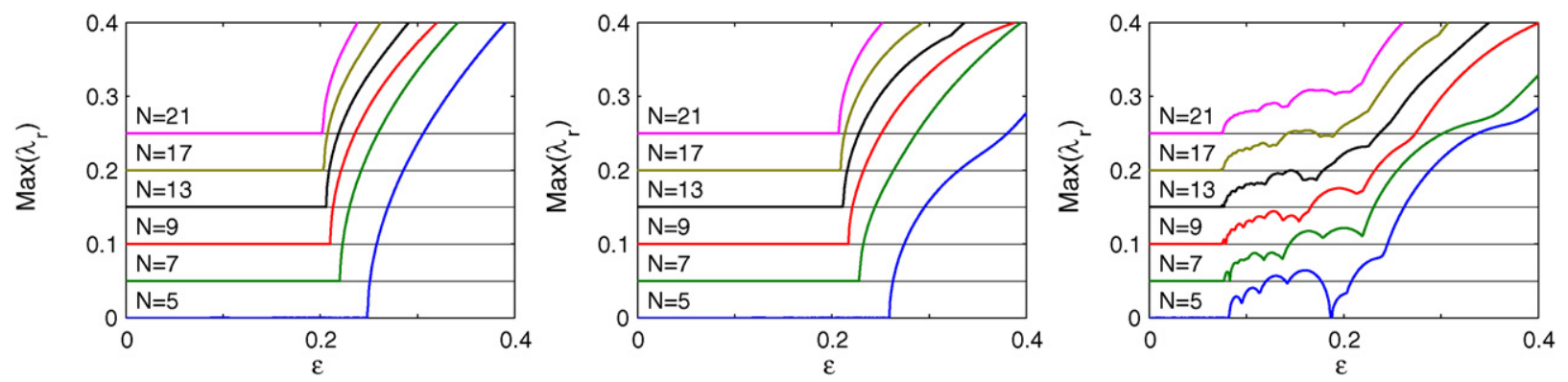

Fig. 9. Lattice size effects on the most unstable eigenvalue for some of the $2 \mathrm{D}$ extended nonlinear structures. The curves depict the most unstable eigenvalue as a function of the coupling strength for different lattice sizes $N \times N$ as indicated (curves are progressively up-shifted by 0.05 for visibility). The left, middle and right panels correspond, respectively, to the $\mathrm{X}-$, Y-, and Z-waves (configurations $2 \mathrm{a}, 2 \mathrm{~b}$, and $3 \mathrm{~b}$, respectively).
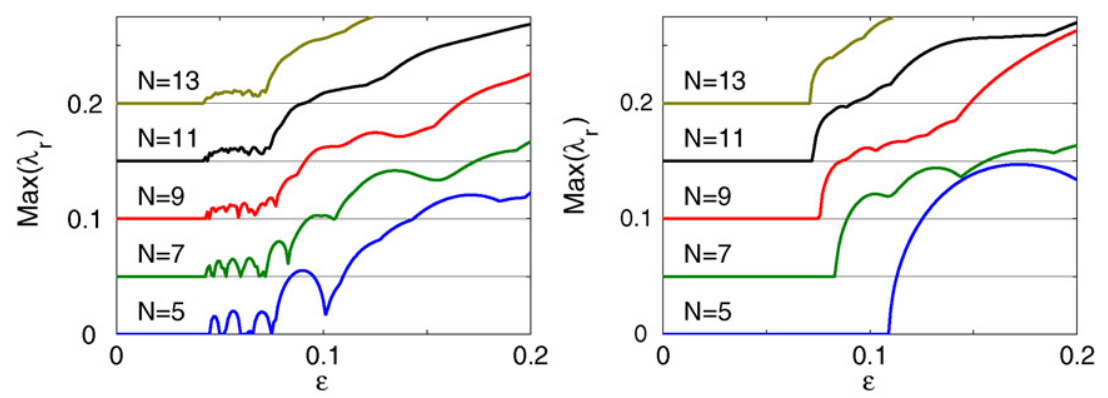

Fig. 10. Lattice size effects on the most unstable eigenvalue for some of the $3 \mathrm{D}$ extended nonlinear structures. The curves depict the most unstable eigenvalue as a function of the coupling strength for different lattice sizes $N \times N \times N$ as indicated (curves are progressively up-shifted by 0.05 for visibility). The left and right panels correspond, respectively, to configurations $4 \mathrm{a}$ and $4 \mathrm{~b}$.

"gaps" in this case within the continuous spectrum that allow for the temporary restabilization of complex eigenvalue quartets.

To conclude this section, we remark that the theoretical bifurcation analysis of the stability of the tile and stone configurations gives excellent qualitative and good quantitative approximations for the full stability of the configurations of interest, as the above results indicate. This then leads us to construct the corresponding ENS configurations by extending the tiles/stones through appending their copies with alternating phases. This is a general procedure that should work for any ENS, provided that the underlying building block (the tile or stone) is dynamically stable.

\section{Dynamics}

In this section we directly examine the evolution of various states, in order to investigate in more detail the dynamical development of the instability. The value $\epsilon=0.5$ was chosen for the coupling strength, which, as observed in the previous section, is well above the instability threshold in all the cases examined. In the simulations of Eq. (1), a small perturbation is added to the initial conditions (except for the case of configuration 3a) in order to initiate the onset of the instability.

Again, the presentation of our numerical results is uniform for all the cases considered in Figs. 11-14. We show the instability development by displaying four snapshots of contour plots of the squared absolute value of the solution. The first (top left) panel is always chosen at some time well before total destruction-typically, when the instability starts to manifest itself. The second and third (top right and bottom left) panels show the dynamics at moments of time when the instability develops. Finally, the fourth (bottom right) panel shows the dynamics after a longer time has elapsed, to illustrate the outcome. In all the configurations, we can observe the growth of the oscillatory instability at the initial stage. This finally seems to give rise, both in 2D and in 3D lattices, to an apparently chaotic configuration where the ENS completely breaks up in fragments dispersed throughout the lattice. This generic feature may be caused by excitation of numerous unstable modes of the configurations for this value of $\epsilon$, taken deep in the instability region (typically, after an ENS becomes unstable, numerous additional destabilizing bifurcations occur at larger values of $\epsilon$ ). 

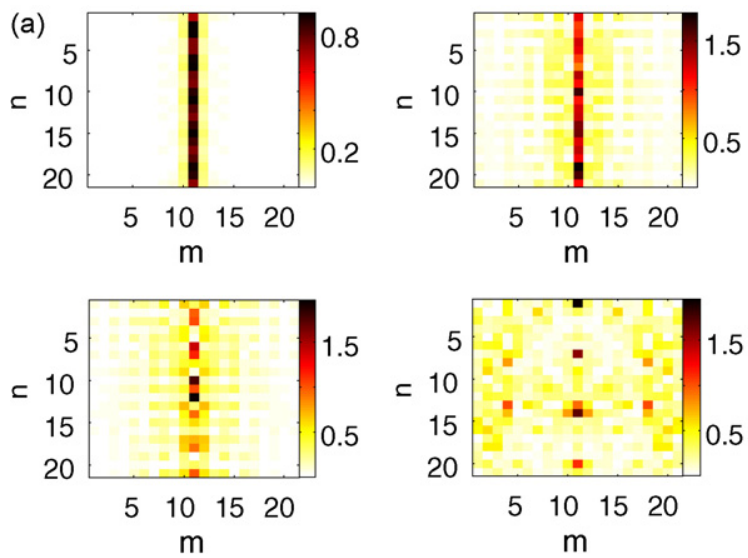
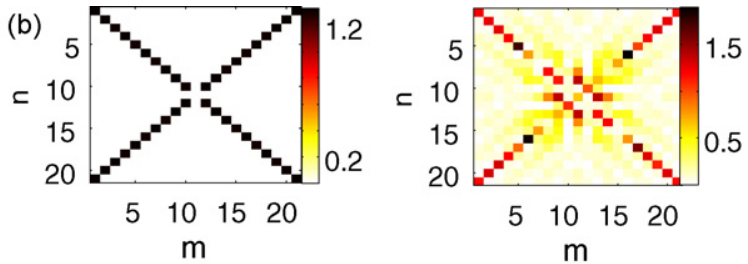
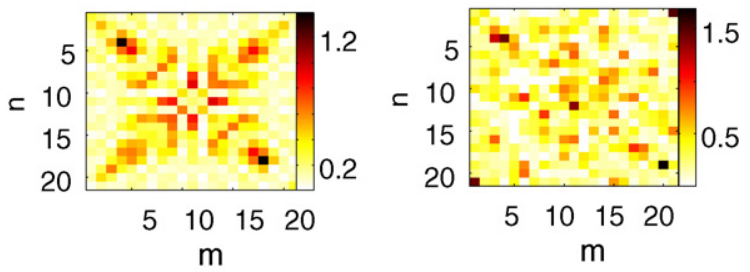

Fig. 11. (a) These panels display the evolution of $|u|^{2}$ for configuration 1 . The panels pertain to $t=20,32,33,50$. (b) The evolution of configuration 2a (X-waves). The panels pertain to $t=15,26,27,40$.
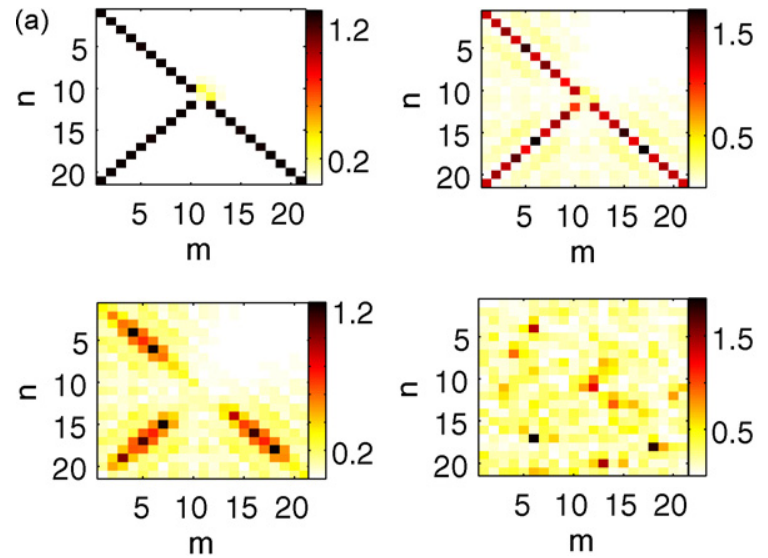
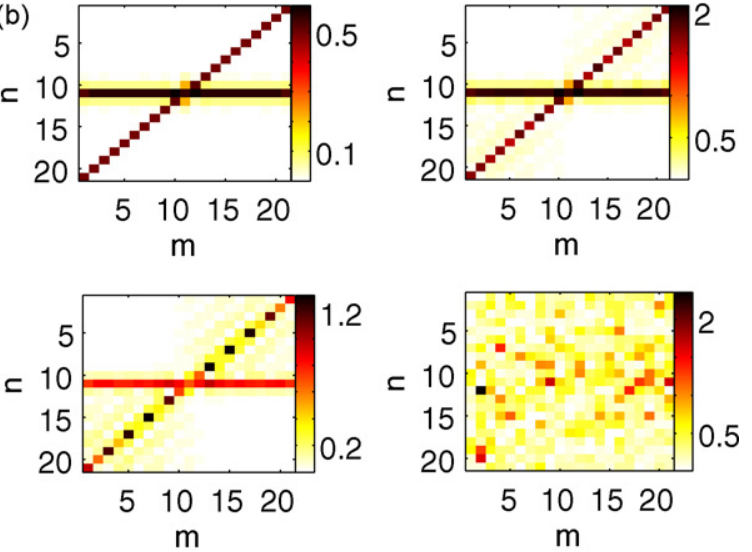

Fig. 12. (a) The evolution of configuration $2 \mathrm{~b}$ (Y-waves) at times $t=15,26,27,50$. (b) The evolution of configuration $2 \mathrm{c}$ (skewed X-waves) at times $t=5,19,20,40$.
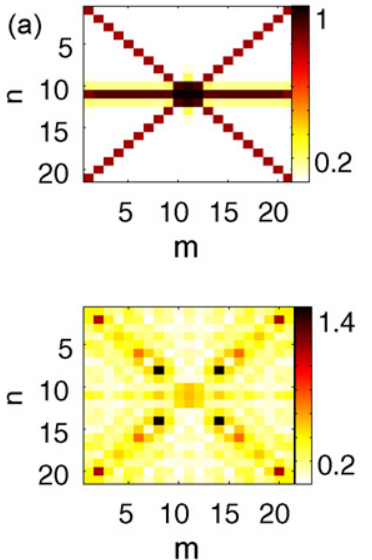
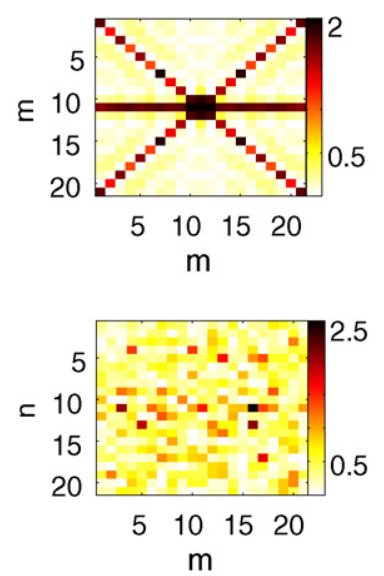
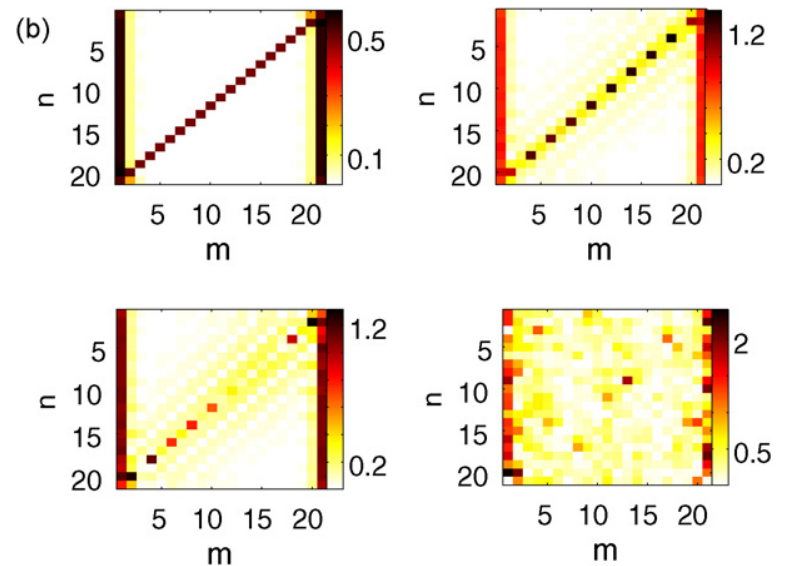

Fig. 13. (a) Same as above, but for configuration $3 \mathrm{a}$ at $t=40,79,80,100$. (b) The same as before, for configuration $3 \mathrm{~b}$ at $t=5,20,21,40$. 

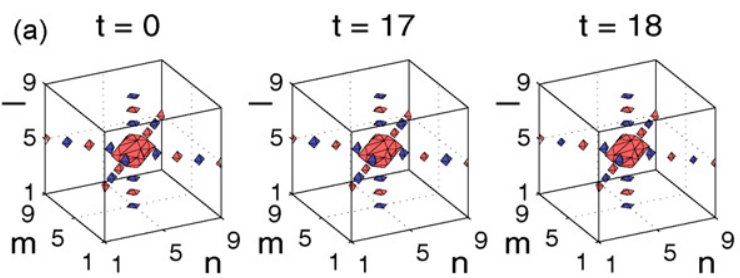

(b)
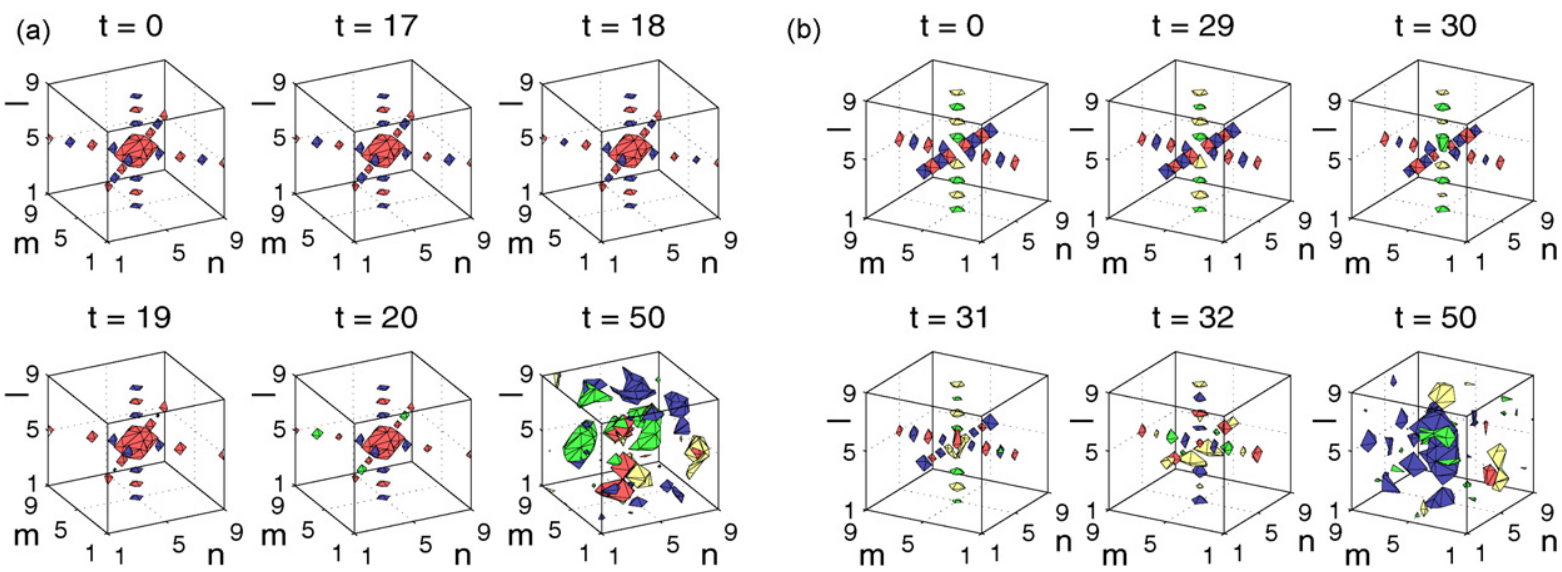

Fig. 14. Time evolution of the unstable 3D configurations (a) $4 \mathrm{a}$ and (b) $4 \mathrm{~b}$ at the times indicated. Each configuration is depicted by using iso-level contours with (i) level contours corresponding to $\operatorname{Re}\left(u_{m, n, l}\right)= \pm 0.5 \operatorname{Max}\left(\left|u_{m, n, l}\right|\right)$ are shown in blue and red (dark gray and gray, in the black-andwhite version), respectively, and (ii) imaginary contours, $\operatorname{Im}\left(u_{m, n, l}\right)= \pm 0.5 \mathrm{Max}\left(\left|u_{m, n, l}\right|\right)$, are shown by green and yellow (light and very light gray, in the black-and-white version) hues, respectively. For a better comparison between the different times, the displayed solutions have been dephased by multiplying them by $\exp (-i \Lambda t)$ and thus eliminating the trivial evolution due to $\exp (i \Lambda t)$. (For interpretation of the references to color in this figure legend, the reader is referred to the web version of the article.)

\section{Conclusion}

In this paper, we have presented a general framework for studying discrete multidimensional extended nonlinear structures (ENSs) and their cores, named "tiles" and "stones", in the 2D and 3D lattice, respectively. Our approach is based on analyzing the existence and stability properties of the corresponding cores. When we found the cores to be stable, we proceeded by extending them into full ENSs configurations by appropriately phase-alternating excited sites at the ends of each core. The analytical predictions were found to agree well with the results of the numerical simulations of the destabilizing bifurcations near the anti-continuum limit. Thus, we have provided a good guide to show what structures could persist as stable ENSs. Naturally, since the relevant patterns could not persist up to the continuum limit, we always found the threshold for their destabilization at finite values of the lattice coupling strength. In the unstable cases, direct numerical simulations were used to showcase the dynamical development of the instability. The latter was observed to typically cause total destruction of the pattern.

It would be of particular interest to extend these results to more realistic settings, in particular those suggested by the experimental results on nonlinear waveguide arrays of Ref. [13]. More generally, it is relevant to generalize the analysis for sign-indefinite Laplacians, both in the quasi-discrete or in the fully discrete case (although the latter one is less directly relevant to the present applications).

\section{Acknowledgments}

P.G.K. acknowledges support from NSF-CAREER, NSF-DMS-0505663 and NSF-DMS-0619492. The work of B.A.M. was in part supported by the Israel Science Foundation through the Center-of-Excellence Grant No. 8006/03, and the German-Israel Foundation through Grant No. 149/2006. R.C.G. acknowledges support from NSF-DMS0505663. The work of D.J.F. was partially supported by the Special Account for Research Grants of the University of Athens.

\section{References}

[1] G.L. Alfimov, V.A. Brazhnyi, V.V. Konotop, On classification of intrinsic localized modes for the discrete nonlinear schrödinger equation, Physica D 194 (2004) 127-150.

[2] V.A. Brazhnyi, V.V. Konotop, Theory of nonlinear matter waves in optical lattices, Mod. Phys. Lett. B 18 (2004) 627-651; O. Morsch, M. Oberthaler, Dynamics of Bose-Einstein condensates in optical lattices, Rev. Mod. Phys. 78 (2006) 179-215; P.G. Kevrekidis, D.J. Frantzeskakis, Pattern forming dynamical instabilities of Bose-Einstein condensates, Mod. Phys. Lett. B 18 (2004) 173-202. 
[3] R. Carretero-González, P.G. Kevrekidis, B.A. Malomed, D.J. Frantzeskakis, Three-dimensional nonlinear lattices: from oblique vortices and octupoles to discrete diamonds and vortex cubes, Phys. Rev. Lett. 94 (2005), 203901-1-203901-4.

[4] Z. Chen, H. Martin, E.D. Eugenieva, J. Xu, A. Bezryadina, Anisotropic enhancement of discrete diffraction and formation of two-dimensional discrete-soliton trains, Phys. Rev. Lett. 92 (2004), 143902-1-143902-4.

[5] D.N. Christodoulides, N.K. Efremidis, P. Di Trapani, B.A. Malomed, Bessel X waves in two- and three-dimensional bidispersive optical systems, Opt. Lett. 29 (2004) 1446-1448.

[6] D.N. Christodoulides, F. Lederer, Y. Silberberg, Discretizing light behaviour in linear and nonlinear waveguide lattices, Nature 424 (2003) 817-823;

S. Yu, G.P. Kivshar, Agrawal, Optical Solitons: From Fibers to Photonic Crystals, Academic Press, San Diego, 2003.

[7] J.W. Fleischer, G. Bartal, O. Cohen, O. Manela, M. Segev, J. Hudock, D.N. Christodoulides, Observation of vortex-ring "discrete" solitons in 2D photonic lattices, Phys. Rev. Lett. 92 (2004), 123904-1-123904-4.

[8] J.W. Fleischer, G. Bartal, O. Cohen, T. Schwartz, O. Manela, B. Freedman, M. Segev, H. Buljan, N.K. Efremidis, Spatial photonics in nonlinear waveguide arrays, Opt. Express 13 (2005) 1780-1796.

[9] M. Golubitsky, D.G. Schaeffer, I. Stewart, Singularities and Groups in Bifurcation Theory, vol. 1, Springer-Verlag, New York, 1985.

[10] M. Johansson, Yu.S. Kivshar, Discreteness-induced oscillatory instabilities of dark solitons, Phys. Rev. Lett. 82 (1999) 85-88.

[11] P.G. Kevrekidis, J. Gagnon, D.J. Frantzeskakis, B.A. Malomed, X.Y.Z. Waves, Extended structures in nonlinear lattices, Phys. Rev. E 75 (2007), 016607-1-016607-6.

[12] P.G. Kevrekidis, K.O. Rasmussen, A.R. Bishop, The discrete nonlinear Schrödinger equation: a survey of recent results, Int. J. Mod. Phys. B 15 (2001) 2833-2900.

[13] Y. Lahini, E. Frumker, Y. Silberberg, S. Droulias, K. Hizanidis, R. Morandotti, D.N. Christodoulides, Discrete X-wave formation in nonlinear waveguide arrays, Phys. Rev. Lett. 98 (2007), 023901-1-023901-4;

M. Heinrich, A. Szameit, F. Dreisow, R. Keil, S. Minardi, T. Pertsch, S. Nolte, A. Tünnermann, F. Lederer, Observation of three-dimensional discrete-continuous X waves in photonic lattices, Phys. Rev. Lett. 103 (2009) 113903-1-113903-4.

[14] C. Lou, X. Wang, J. Xu, Z. Chen, J. Yang, Nonlinear spectrum reshaping and gap-soliton-train trapping in optically induced photonic structures, Phys. Rev. Lett. 98 (2007), 213903-1-213903-4.

[15] J.Y. Lu, J.F. Greenleaf, Nondiffracting X-waves: exact solutions to free-space scalar wave equation and their finite aperture realizations, IEEE Trans. Ultrason. Ferroelectr. Freq. Control 39 (1992) 19-31;

P. Di Trapani, G. Valiulis, A. Piskarskas, O. Jedrkiewicz, J. Trull, C. Conti, S. Trillo, Spontaneously generated X-shaped light bullets, Phys. Rev. Lett. 91 (2003), 093904-1-093904-4;

C. Conti, S. Trillo, P. Di Trapani, G. Valiulis, A. Piskarskas, O. Jedrkiewicz, J. Trull, Nonlinear electromagnetic X waves, Phys. Rev. Lett. 90 (2003), 170406-1-170406-4.

[16] D.N. Neshev, T.J. Alexander, E.A. Ostrovskaya, Y.S. Kivshar, H. Martin, I. Makasyuk, Z. Chen, Observation of discrete vortex solitons in optically induced photonic lattices, Phys. Rev. Lett. 92 (2004), 123903-1-123903-4.

[17] D.E. Pelinovsky, P.G. Kevrekidis, D.J. Frantzeskakis, Stability of discrete solitons in nonlinear schrödinger lattices, Physica D 212 (2005) $1-19$.

[18] D.E. Pelinovsky, P.G. Kevrekidis, D.J. Frantzeskakis, Persistence and stability of discrete vortices in nonlinear Schrödinger lattices, Physica D 212 (2005) 20-53.

[19] M. Peyrard, Nonlinear dynamics and statistical physics of DNA, Nonlinearity 17 (2004) R1-R40.

[20] J. Yang, I. Makasyuk, A. Bezryadina, Z. Chen, Dipole and quadrupole solitons in optically induced two-dimensional photonic lattices: Theory and experiment, Stud. Appl. Math. 113 (2004) 389-412. 